\title{
Protée
}

\section{Du sens aux sens : les représentations mentales dans l'acte de lecture}

\section{Sylvain Brehm}

Volume 39, numéro 2, automne 2011

URI : https://id.erudit.org/iderudit/1007173ar

DOI : https://doi.org/10.7202/1007173ar

Aller au sommaire du numéro

Éditeur(s)

Département des arts et lettres - Université du Québec à Chicoutimi

\section{ISSN}

0300-3523 (imprimé)

1708-2307 (numérique)

Découvrir la revue

Citer cet article

Brehm, S. (2011). Du sens aux sens : les représentations mentales dans l'acte de lecture. Protée, 39(2), 107-112. https://doi.org/10.7202/1007173ar
Résumé de l'article

Le statut des représentations mentales dans la lecture n'a fait l'objet que de peu d'études approfondies. Les travaux fondateurs menés en théorie littéraire insistent surtout sur la dimension conceptuelle de ces représentations. Les sciences cognitives, de leur côté, s'intéressent également aux modalités de la compréhension des textes, mais ne prennent en compte ni la dimension esthétique de ces derniers ni la singularité de l'acte de lecture. Nous proposons d'instaurer un dialogue interdisciplinaire afin de montrer que les représentations mentales contiennent des composantes aussi bien sensorielles qu'émotionnelles et conceptuelles. 


\title{
DU SENS AUX SENS: LES REPRÉSENTATIONS MENTALES DANS L'ACTE DE LECTURE
}

\author{
SYLVAIN BREHM
}

S'il est trivial d'affirmer que l'acte de lecture repose sur la formation de représentations mentales, on peut s'étonner que leur nature et leur rôle fassent l'objet de peu de recherches approfondies en études littéraires, hormis quelques travaux récents (notamment Gervais, 2007). De ce point de vue, la réflexion que Wolfgang Iser consacre à la lecture (Iser, 1976) paraît particulièrement intéressante, malgré les critiques justifiées qu'elle a suscitées, car elle vise à mettre au jour les modalités fondamentales du processus d'appariement entre les signes verbaux et les représentations mentales. Iser, cependant, accorde un statut particulier à ces dernières en privilégiant leur dimension sémantique (conceptuelle) au détriment de leur aspect iconique et, plus largement, sensoriel (perceptuel).

Cette position ambiguë témoigne d'une prévention à l'égard des images mentales, qui se manifeste aussi dans les recherches en sciences cognitives depuis la publication des travaux fondateurs de Kintsch et Van Dijk (1978), puis de Johnson-Laird (1983).

Un tel constat nous invite à proposer une analyse de la nature et de la fonction des représentations mentales formées durant la lecture, en tenant compte aussi bien des propositions avancées par des théoriciens de la littérature que des résultats des recherches menées dans le domaine des neurosciences. Ce dialogue interdisciplinaire nous paraît non seulement utile, mais également nécessaire et fécond, en ce qu'il permet de poser un regard novateur sur l'acte de lecture. Pour ce faire, nous commencerons par examiner les modalités et les composantes de la production des représentations mentales. Puis, nous montrerons, en particulier à l'aide des résultats de travaux en neuro-imagerie, que l'acte de lecture requiert la mobilisation de processus perceptifs et affectifs, et qu'il permet aussi de construire des représentations nouvelles à partir de simulacres d'expériences sensorielles et émotionnelles.

\section{L'élaboration d'une représentation mentale}

Étonnamment, alors que le terme «imaginaire» se retrouve fréquemment dans le discours des théories littéraires, les images mentales elles-mêmes n'ont fait l'objet que de très peu d'études approfondies. L'influence de la phénoménologie husserlienne et de la psychologie de la forme (Gestalttheorie) amène néanmoins Iser à concevoir l'acte de lecture comme un processus de construction d'une forme déterminé par une succession de perspectives textuelles qui dévoilent les aspects de l'objet visé. La représentation imaginaire résulte ainsi d'une série de synthèses produites grâce à ce «point de vue mobile». La notion de "perspective» rend bien compte de ce qui détermine le rapport à l'objet:

[...] notre accès au monde fictionnel se fait toujours à travers

l'aspectualité sous laquelle il est présenté, aspectualité qui est enchâssée dans les modalités spécifiques de l'amorce mimétique qui induit l'attitude représentationnelle. (Schaeffer, 1999: 228)

Toutefois, la relation entre la signification du texte et la représentation élaborée par le lecteur revêt un caractère particulier selon Iser, car le texte ne fournit au lecteur que les conditions nécessaires à l'élaboration d'une représentation mentale. En effet, en raison de la diversité et de la complexité des objets visés par les œuvres littéraires, I'imagination ne se limite pas à convoquer un matériau issu d'expériences préalables. De plus, rien ne garantit que le savoir constituant le fondement 
des représentations élaborées au cours de la lecture soit délivré de manière linéaire et continue. Dans bien des cas, le texte érige des perspectives multiples, convergentes ou divergentes, qui contraignent le lecteur à restructurer les configurations produites sur la base d'un rapport dialectique entre les informations conservées en mémoire (rétentions) et les anticipations formées (protentions). La configuration représentative résulte alors d'une synthèse dynamique fondée sur la relation entre les rétroactions et les attentes: "chaque nouvelle association établie entre facettes isolées donne lieu à une image mentale à laquelle le lecteur réagit par une nouvelle image dès lors que de nouveaux aspects doivent s'y intégrer » (Iser, 1985 : 251). Le caractère dynamique des synthèses représentatives procède, en somme, de l'actualisation progressive et constante de la forme élaborée au cours de la lecture à partir du matériau sémiotique offert par le texte.

Bien que les théories des modèles (modèle de situation de Kintsch et Van Dijk, modèle mental de Johnson-Laird) s'inscrivent dans un horizon conceptuel différent et qu'elles n'aient pas la même visée que la démarche d'Iser, plusieurs points communs méritent d'être signalés. Rappelons, dans un premier temps, les principes fondamentaux des modèles mentaux ${ }^{1}$ : la compréhension d'un texte met en œuvre trois niveaux distincts de représentation (structure de surface, représentation propositionnelle, modèle de situation ou modèle mental). La représentation propositionnelle est produite, automatiquement et involontairement, à partir du traitement de l'information textuelle (structure de surface). Elle se présente comme un enchaînement de propositions et demeure étroitement liée aux contraintes lexicales et syntaxiques de la langue. En revanche, le dernier niveau, celui du modèle mental, n'est plus associé à une représentation linguistique; il forme une structure, globale et cohérente, analogue à l'état du monde visé par le texte.

Selon Iser, on l'a vu, le lecteur est amené à construire la signification du texte grâce aux perspectives que ce dernier lui offre. De la même manière, le modèle mental est multidimensionnel en ce qu'il résulte de l'intégration d'éléments associés au temps, à l'espace, à l'enchaînement des actions et au(x) personnage(s) (Johnson-Laird, 1983). Les recherches ultérieures vont mettre au jour la nécessité de prendre en compte d'autres dimensions, telles que les émotions et le genre (gender) des protagonistes, et mesurer la capacité d'un lecteur empirique à intégrer effectivement cette pluralité de composantes textuelles (la question ne se posant pas pour Iser, puisque son «lecteur implicite» est une pure stratégie textuelle).
La représentation formée au cours de la lecture est soumise à un processus constant de révision et d'actualisation, car sa production s'amorce au seuil de la lecture et est enrichie en fonction des informations disponibles et des inférences du lecteur:

[l]e modèle commence à être élaboré dès le début de la lecture (audition) du texte et il est ensuite progressivement enrichi et modifié ; le modèle formé à un moment donné de la lecture sert de guide à l'élaboration du modèle généré à partir de la suite $d u$ texte. (Ehrlich et Tardieu, 1993: 50)

Morton Ann Gernsbacher (1990) précise les modalités de I'actualisation du modèle mental en affirmant que plus le texte est complexe, plus la représentation mentale produite en cours de lecture est soumise à des procédures de révision et de correction sur la base aussi bien de ce qui est conservé en mémoire que des attentes qui se forment à chaque instant. Ainsi, dans le «modèle de construction de structure» (structure building framework), la production de structures mentales est amorcée à partir d'une information qui sert de fondement (foundation) et à laquelle sont intégrées toutes les données ultérieures dès lors qu'elles ne remettent pas en cause la cohérence de la structure produite jusque-là (mapping). Lorsqu'un changement de thème ou de personnage, par exemple, survient, une nouvelle représentation est créée (shifting). Au cours de la lecture, plusieurs sous-structures sont ainsi produites et s'agrègent au sein d'une macrostructure qui tient lieu de représentation du texte.

Un constat s'impose: toutes ces modélisations de l'acte de lecture se concentrent essentiellement sur les modalités de l'actualisation de la signification d'un texte. Or, si l'accès au monde fictionnel est incontestablement médiatisé par un ensemble de structures sémantiques, la lecture, en tant qu'expérience esthétique, revêt des dimensions affective et sensorielle, tout autant que cognitive. Il apparaît essentiel, à cet égard, d'examiner la nature des éléments qui forment les représentations mentales.

\section{Les composantes de la représentation}

Compte tenu de l'usage de nombreuses métaphores optiques dans la théorie d'Iser, il n'y a, a priori, rien d'étonnant à ce que ce dernier affirme que «l'image est le mode d'apparition de l'objet littéraire» (1985: 254). Un examen attentif de ses propositions révèle, cependant, qu'il privilégie la dimension conceptuelle des représentations mentales ${ }^{2}$. Celles-ci « véhiculent tantôt des images, tantôt des significations» (ibid. : 265), mais comme le précise Ellen J. Esrock, 
[...] bien que ces constructions hybrides contiennent une composante iconique, celle-ci ne peut pas être identifiée de manière spécifique, même lorsqu'elle est analysée et portée à la conscience en raison de ce qu'Iser appelle la «nature transitoire de l'image » et "sa fonction vitale de fusion ». 3

(1994: 29-30; notre traduction)

La dimension visuelle de l'image mentale s'en trouve considérablement réduite:

[...] lorsque, dans le roman, le personnage nous est décrit de façon détaillée, [...] en général nous ne lisons pas cette description en tant que description pure et simple du personnage, mais nous nous demandons ce que cette description doit signifier. (Iser, 1985: 250)

Dans Volkswagen blues de Jacques Poulin, par exemple, quelques caractéristiques de Pitsémine nous sont livrées dès I'incipit: la Métisse est une "grande fille maigre», ses cheveux sont «noirs comme du charbon et nattés en une longue tresse qui lui descend [...] au milieu du dos» (1984: 9). Ces informations, intégrées dans une configuration signifiante, nous permettent d'actualiser les premiers traits représentatifs de la jeune femme: la maigreur de son corps annonce son aspect androgyne et sa natte de cheveux noirs signale ses origines amérindiennes. Toutefois, avec quelle certitude peut-on affirmer que l'ensemble des lecteurs décèle, d'emblée, l'importance de tels éléments et décide de les conserver en mémoire en attente d'autres indices pertinents? De plus, si la valeur signifiante de ces informations paraît indiscutable, en quoi implique-t-elle nécessairement le rejet de leur dimension iconique?

Les travaux fondateurs sur les modèles mentaux n'apportent guère de réponses à ces questions, puisqu'ils n'accordent qu'un rôle résiduel aux images mentales. Cela tient sans doute, du moins en partie, à l'influence de la cybernétique, de I'informatique et de l'intelligence artificielle sur les premières générations de recherches en sciences cognitives (JohnsonLaird, 1988). Animés par la volonté de modéliser les processus universels de traitement de l'information, les psychologues et les psycholinguistes mettent l'accent sur la compréhension et la mémorisation de textes narratifs ou descriptifs généralement brefs et globalement univoques. La dimension esthétique de ces derniers n'est nullement prise en compte, pas plus que la variabilité des interprétations ou la singularité de l'investissement cognitif et imaginaire de chaque lecteur:

Jusqu'à maintenant la psychologie a étudié la signification objective des choses et des événements et a délaissé l'étude de leur signifi- cation qui dépendrait des affects, des émotions et des motivations des individus. (Syssau, 2006: 66)

Ce clivage se manifeste dans la distinction opérée par JohnsonLaird entre «modèle mental » et « image mentale», cette dernière ne représentant qu'un aspect partiel et subjectif d'un savoir pur: "les modèles mentaux sont des structures analogues au monde, et les images [...] sont les corrélats perceptifs des modèles à partir $d^{\prime}$ un point de vue particulier ${ }^{4}$ (Johnson-Laird, 1983: 165 ; notre traduction). À ce titre, si un modèle mental peut contenir des images, il ne se confond pas avec elles. Comme dans la théorie isérienne, la représentation mentale du monde se réduit à ses aspects conceptuels.

En somme, bien qu'ils s'inscrivent dans des champs de recherche radicalement distincts, les travaux menés, jusqu'au début des années 1990, en études littéraires et en sciences cognitives s'accordent sur plusieurs points, notamment sur le statut et la fonction des représentations mentales produites lors de la lecture. Même si des cogniticiens comme Michel Denis (1989) reconnaissent le rôle, optionnel mais néanmoins important, des images au cours de la lecture, elles conservent un rôle négligeable dans les théories des modèles mentaux, qui font pourtant aujourd'hui consensus en psychologie cognitive.

\section{Du sens aux sens}

La compréhension plus fine, grâce aux techniques de neuro-imagerie, des interrelations entre les différents processus cognitifs, de même que l'intérêt grandissant accordé au lecteur - sujet et acteur d'une expérience esthétique -, ont conduit à remettre en question ces conceptions exclusives.

Gilles Thérien, par exemple, souligne très justement que toute lecture littéraire est « un travail d'imagination » et de construction permettant à un sujet « $d^{\prime}$ investir, dans un mouvement qui va de l'intérieur vers l'extérieur, [s]es images sous les mots du texte. » (2007 : 223). Christine Baron, pour sa part, souligne le rôle essentiel de l'image aussi bien dans l'écriture que dans la lecture de l'œuvre d'un romancier comme Italo Calvino:

[...] l'image est tantôt "en amont" de l'écriture comme une sorte d'input visuel, tantôt en aval sous une forme prospective, mais toujours indissociable d'une pré-formation d'un concept $d u$ monde, d'un modèle auquel elle prêterait sa visualité, plutôt que sa visibilité. (2007: 23)

De tels propos rappellent ceux de Pierre Ouellet, pour qui la littérature est toujours, par essence, «hybride de figures sensibles 
et de pures notions » (2000: 9). Si l'univers narratif se dérobe à toute saisie sensorielle directe,

[l]a littérature nous fait vivre une expérience perceptive médiate dont l'objet est imaginaire, puisque construit à partir d'images mentales résultant de notre double connaissance de la langue et du monde. (Ibid.)

Ces réflexions invitent à dépasser le clivage systématique entre perception et cognition, et à reconsidérer le rôle essentiel de l'image en tant que support de la figuration du sens.

Un questionnement similaire est apparu dans les sciences cognitives depuis quelques années. Pour les théories de la cognition incarnée (embodied theories of language), en particulier, les connaissances sont maintenues en mémoire à long terme sous une forme amodale (conceptuelle), mais aussi sous une forme modale (Barsalou, 1999). Autrement dit, toutes les sensations extéroceptives (vision, audition, etc.) et proprioceptives (frisson, crispation, etc.) liées à cette connaissance sont également conservées en mémoire. En vertu de cette interrelation, l'évocation d'une situation ou d'un objet particuliers est susceptible d'éveiller des traces mnésiques de perceptions ou d'émotions associées à des expériences antérieures (Syssau, 2006: 49). La lecture d'un extrait du Pavillon des miroirs, de Sergio Kokis, va nous permettre d'illustrer ce phénomène:

Ravagé par les feux et les fourmis, le paysage tropical devient désert. Les arbres dénudés et noircis restent sur place, tels des squelettes qui crient sous le soleil. La seule protestation visible. Le vert devient de plus en plus sale, plutôt gris, et l'oxyde de fer sous toutes ses teintes assomme le regard. Les habitants aussi se plient à cette hiérarchie des ocres : leur peau, les vêtements sales, les cuirs crus des chapeaux et des sandales, jusqu'au violet tête de mort autour des orbites des enfants. Au matin, le contraste de tous ces teints de terre avec le bleu du ciel est saisissant, et les nids de fourmis éclatent alors comme des taches de sang. (1994: 260)

Parmi les procédés rhétoriques dont use Kokis pour brosser ce tableau sordide et suggestif, l'hypotypose confère au paysage décrit le pouvoir d' «assomme[r] le regard » et de frapper l'imagination. L'auteur, qui rappelons-le est aussi peintre, agence un dispositif scripto-visuel particulièrement efficace. Les rapports antithétiques entre certains éléments descriptifs participent de cette volonté de produire des contrastes violents: I'aridité du désert succède brutalement à la luxuriance du paysage tropical, le bleu du ciel s'oppose à l'ocre de la terre et des visages (I'ocre est composé d'orangé - mélange de jaune et de rouge-, couleur complémentaire du bleu). La visualité de la scène est renforcée par le choix des termes utilisés par le narrateur pour désigner, sans entrer dans les nuances, les différents registres chromatiques: noir, vert, gris, violet, bleu, etc.

Enfin, les comparaisons et les métaphores favorisent le "déploiement iconique du sens dans I'imaginaire» (Ricœur, 1975 : 267) en établissant des analogies d'où surgissent des images nouvelles investies d'une forte charge affective (associée à la souffrance et à la morbidité) : silhouettes décharnées des arbres calcinés semblables à celles de squelettes figés dans la douleur, activité bouillonnante des fourmilières évoquant les plaies ouvertes de la terre brûlée par le soleil.

Le travail d'écriture, loin d'opacifier le rapport du lecteur à une réalité qui peut lui apparaître totalement étrangère, stimule, au contraire, l'imagination de ce dernier grâce à la présence et à la richesse de plusieurs indices de figurabilité. Ces composantes textuelles à visée référentielle (en particulier les embrayeurs sensoriels et les éléments descriptifs évoqués plus haut) médiatisent le rapport du lecteur au monde fictionnel et déterminent, dans une certaine mesure, la forme que revêt « la rencontre intime des images qui habitent le lecteur et des mots du texte» (Thérien, 2007 : 205). L'activité imaginative, en effet, peut aisément s'appuyer sur un ensemble d'images issues de perceptions antérieures ou de souvenirs d'anciennes lectures. À ce titre, le dispositif textuel instaure les conditions propices à l'expérience d'une quasi-perception, que Ricœur nomme voir comme: «le voir comme mis en ouvre dans l'acte de lire assure la jonction entre le sens verbal et la plénitude imagière » (ibid. 270). Aussi, le « voir-comme», en tant qu'il conduit à la figuration du sens, constitue, comme sa représentation sémantique, l'un des aspects de sa concrétisation.

Les découvertes les plus récentes en neurophysiologie confirment cette idée et accréditent la thèse que l'activité de lecture ne mobilise pas seulement les aires cérébrales impliquées dans le traitement d'informations purement abstraites: «les représentations des situations décrites à l'aide du matériau linguistique sont construites par le lecteur à partir de représentations sensorielles et motrices fondamentales » 5 (Speer, 2009: 997; notre traduction). Le problème qui semble se poser, néanmoins, tient à la difficulté à expliquer qu'on puisse mobiliser et construire des représentations mentales même lorsque des textes évoquent une situation qui nous est inconnue.

Speer et ses collaborateurs ont montré que la production des représentations mentales durant la lecture repose aussi sur la formation de simulacres de données perceptives et sensibles. 
Ce phénomène s'appuierait notamment sur I'existence de neurones-miroirs (Rizzolatti, 2004), stimulés chez un individu observant, par exemple, quelqu'un d'autre accomplir une tâche ou manifester une émotion exactement comme si lui-même agissait ou éprouvait cet affect. La complexité de l'acte de lecture appelle incontestablement à nuancer la portée de cette découverte, notamment car «l'imagerie mentale est affectée à la fois par les facultés du lecteur et les caractéristiques du texte 6 (Long, 1989: 353 ; notre traduction). En effet, chaque œuvre littéraire structure ses propres conditions d'accès au monde fictionnel, en particulier grâce au nombre et au caractère plus ou moins déterminé des indices de figurabilité qu'il contient. De même, il est nécessaire de tenir compte de la compétence imaginative de chaque sujet liée, d'une manière générale, à des facteurs biologiques, mais aussi, d'une manière plus spécifique, au type de textes lus: une compétence lexicale insuffisante ou la confrontation à un référentiel fictionnel totalement inconnu laissent le lecteur relativement démuni.

Si ces réserves sont légitimes, force est néanmoins de reconnaître que I'on peut très bien visualiser la scène évoquée dans l'extrait du roman de Kokis sans être jamais allé dans le Nordeste brésilien. Ce phénomène est parfaitement cohérent avec les résultats d'expérimentations qui ont montré que, en mobilisant des représentations sensorielles, affectives et motrices préalables, «les lecteurs construisent des simulations des situations qu'ils lisent dans les textes, et que ce processus est similaire à celui de se remémorer des expériences antérieures ou $d^{\prime}$ imaginer des situations fictives " 7 (Speer, 2009: 997). Notons que ces nouvelles représentations issues d'expériences virtuelles pourront, ultérieurement, être mobilisées au même titre que des composantes issues d'expériences directes. Ainsi, à défaut de n'avoir jamais vu des dragons ou des licornes, nous convoquons, en lisant des romans de Chrétien de Troyes, des images de ces animaux mythologiques élaborées à partir d'autres romans, ou de films, de jeux vidéo, etc. C'est précisément parce que I'imagination permet la production de configurations composites présentant des aspects sensibles, affectifs et conceptuels que la lecture revêt un caractère heuristique et expérientiel.

En somme, si l'accès à la signification d'un énoncé ne requiert pas nécessairement la formation d'images mentales, rien ne permet de conclure pour autant que ces dernières n'ont qu'une fonction résiduelle ou encore qu'elles sont essentiellement conceptuelles. Au contraire, les avancées récentes des sciences cognitives tendent à réconcilier deux approches que l'on a trop longtemps tenues pour irréconciliables: celle privilégiant I'aspect sémantique de la représentation et celle insistant sur sa dimension figurative. L'orientation des recherches récentes, tant en théorie littéraire qu'en psychologie cognitive et en psycholinguistique, met au jour l'importance de déterminer, $d^{\prime}$ une part, les relations complexes qui se nouent entre les signes verbaux et les représentations qui leur sont appariées dans la conscience du lecteur, et, d'autre part, les liens entre les différentes composantes des représentations mentales.

Ce vaste chantier de réflexion est prometteur non seulement en ce qu'il nous laisse espérer mieux comprendre ce qui détermine notre rapport aux œuvres esthétiques (et, à travers elles, au monde qui en est l'horizon), mais aussi parce qu'il rappelle à quel point la fréquentation des textes littéraires revêt un rôle essentiel dans la formation de chaque individu. Comme le souligne Gilles Thérien, le produit de la lecture est intégré dans des systèmes symboliques qui ont « une valeur référentielle en tant que hiérarchie, systèmes scientifiques, savoirs, pratiques, rituels, idéologies ou imaginaires» (1990: 75). Insister sur la relation privilégiée que la littérature entretient avec ces systèmes symboliques tout en réhabilitant la composante subjective (souvenirs, sensations, émotions, etc.) de l'acte de lecture permet de souligner que ce dernier est « un des principaux lieux de transaction entre le singulier et le collectif » (Thérien, 2007 : 222). À ce titre, la lecture apparaît comme le facteur $d^{\prime}$ une socialisation déterminée à la fois par la rencontre entre le monde du texte et celui du lecteur, et par l'élaboration d'un imaginaire partagé. 


\section{NOTES}

1. Les concepts de modèle de situation et de modèle mental présentent des divergences, mais leurs principes fondamentaux sont les mêmes, de sorte qu'une certaine confusion terminologique apparaît dans les nombreuses études qui font référence à l'un ou à l'autre. Pour notre part, nous utiliserons l'expression «modèle mental » proposée par Johnson-Laird. 2. Il est à noter que dans The Fictive and the Imaginary: Charting : charting literary and anthropology, paru en 1993, Iser n'approfondit malheureusement pas la relation entre l'acte de lecture et l'imaginaire. 3. "although these hybrid constructions contain a pictorial element, this composant cannot be specifically identified, even when formulated and brought to consciousness because of what Iser terms 'the transitory nature of the image' and 'its vital function of fusion'."

4. "mental models $[\ldots]$ are structural analogues of the world, and images [...] are the perceptual correlates of models from a particular point of view."

5. "reader's representations of situations described in language are constructed from basic sensory and motor representations."

6. "the production of imagery is affected both by reader and text characteristics."

7. "[...] readers construct simulations of situations as they read a text, and that this process is similar to recalling previous situations or imagining potential ones."

\section{RÉFÉRENCES BIBLIOGRAPHIQUES}

BARON, C. [2007] : «Imaginaire, visualité, fiction», dans C. Grall (dir.), Récit de fiction et représentation mentale, Mont-Saint-Aignan, Presses des universités du Rouen et du Havre, 11-27.

BARSALOU, L. W. [1999] : «Perceptual Symbol Systems », Behavioral and Brain Sciences, no 22, 577-660.

DenIS, M. [1989] : Image et Cognition, Paris, PUF, coll. "Psychologie d'aujourd'hui ».

EHRLICH, M.-F et H. TARDIEU [1993] : "Modèles mentaux, modèles de situation et compréhension de textes », dans M.-F. Ehrlich, H. Tardieu et M. Cavazze (dir.), Les Modèles mentaux. Approche cognitive des représentations, Paris, Masson, 47-77.

ESROCK, E. J. [1994]: The Reader's Eye. Visual Imaging as Reader Response, Baltimore, Johns Hopkins University Press.
GernSBACHeR, M. A. [1990]: Language Comprehension as Structure Building, Hillsdale, L. Erlbaum.

GeRvaIS, B. [2007] : Figures, lectures. Logiques de l'imaginaire, tome I, Montréal, Le Quartanier, coll. «Erres essais».

ISER, W. [(1976) 1985] : L'Acte de lecture. Théorie de l'effet esthétique, Bruxelles, P. Mardaga.

JOHNSON-LAIRD, P. [1983] : Mental Models: Towards a Cognitive Science of Language, Inference and consciousness, Cambridge, Harvard University Press;

- [1988]: The Computer and the Mind: An Introduction to Cognitive Sciences, Cambridge, Harvard University Press.

KINTSCH, W. et T. A. VAN DIJK [1978]: "Toward a model of text comprehension and production», Psychological Review, vol. 85, no 5, 363-394 KOKIS, S. [1994]: Le Pavillon des miroirs, Montréal, XYZ, coll. "Romanichels".

LONG, S. et alii [1989] : "The Effects of Reader and Text Characteristics on Report on Imagery Reported during and after Reading », Reading Research Quarterly, no 24, 353-372.

OuELLET, P. [2000] : Poétique du regard: littérature, perception, identité, Sillery et Limoges, Septentrion et Pulim, coll. «Les nouveaux cahiers du CELAT ».

POuLIN, J. [1984]: Volkswagen blues, Montréal, Québec/Amérique, coll. "Littérature d'Amérique».

Ricoeur, P. [1975], La Métaphore vive, Paris, Seuil, coll. «Points». Rizzolatti, G. et L. Craighero [2004] : «The Mirror-Neuron System », Annual Review of Neurosciences, no 27, 169-192.

Schaeffer, J.-M. [1999] : Pourquoi la fiction?, Paris, Seuil, coll. «Poétique».

SPEER, N. et alii [2009] : «Reading Stories Activates Neural Representations of Visual and Motor Experiences », Psychological Science, vol. 20 $\mathrm{n}^{\circ} 8,989-999$.

SYSSAU, A. [2006] : «Émotion et cognition», dans N. Blanc. (dir.), Émotion et Cognition. Quand l'émotion parle à la cognition, Paris, In Press, 11-67. THÉRIEN, G. [1990] : «Pour une sémiotique de la lecture», Protée, vol. 18, $\mathrm{n}^{\circ} 2$, printemps, $67-80$;

[(2000) 2007] : "Les images sous les mots », dans B. Gervais et R. Bouvet (dir.), Théories et pratiques de la lecture littéraire, Québec, Presses de l'Université du Québec, 205-223. 